La cohésion et la cohérence discursives

de l'épisode polémique dans

Le destin de Iouri Voronine D'Henriette Jelinek

**Recherche présentée par:

Noha Salah El Din Awad

Maître de conférences(linguistique)

Faculté des Lettres-Université de Ménoufeya 


\section{La cohésion et la cohérence discursives de l'épisode polémique dans Le destin de Iouri Voronine D'Henriette Jelinek}

Après des études de psychologie,Henriette Jelinek ${ }^{1}$ enseigne les mathématiques aux élèves en difficulté.Enseignante remarquable,elle se passionne également pour l'écriture et la littérature.C'est à Raymond Queneau qu'elle doit la découverte de son premier roman La Vache multicolore ${ }^{2}$ publié en $1961 \mathrm{chez}$ Gallimard.Grâce à cette œuvre,Jelinek est parvenue à marquer un public impressionné par le regard véhément et sincère qu'elle porte sur ses personnages.

Depuis 1968,elle abandonne l'enseignement et se consacre à la littérature.Jelinek a publié treize romans dont Portrait d'un séducteur ${ }^{3}$ et Une goutte de poison ${ }^{4}$.Elle a également écrit pour le quotidien Le Monde et Le Matin de Paris, et co-sénarisé le film de Jeanne Moreau L'Adolescente en 1978.

Tout au long de ses romans,Jelinek cherche à disséquer les travers et les passions des personnages.Elle tente,par la suite, de refléter leur univers psychologique et de dévoiler les égarements de leur cœur et de leur esprit.

Éstimée par nombre de ses contemporains,Jelinek"montre qu'elle atteint la pleine maîtrise de son art ${ }^{\prime \prime 5}$ par son dernier roman Le Destin de Iouri Voronine ${ }^{6}$ couronné par le Grand Prix du roman de L'Académie française.Ainsi avons-nous choisi d'étudier ce chef-d'œuvre qui raconte l'histoire de Iouri,un vieux russe qui a émigré à Chicago où il a vécu cinquante ans depuis son arrivée aux États-Unis.Après la mort de sa femme,Iouri,pauvre retraité,a été transporté dans la luxueuse villa de son fils unique Joe à Beverly Hills.

1)JELINEK(H.) est "née en 1923 dans les Landes" et morte "le 3 novembre 2007". http://www.linternaute.com/biographie/henriette-jelinek/biographie/ consulté le 17/03/2009.

2)JELINEK(H.),La Vache multicolore,Paris,Éd.Gallimard,1961.

3)ID, Portrait d'un séducteur,Paris,Éd.Gallimard,1965.

4)ID, Une goutte de poison,Paris,Éd.Ramsay,1987.

5)http://www.alapage.com/-/Fiche/Livres/9782848681160/LIV/le-destin-de-iouri-voron... consulté le 29/03/2009.

6) JELINEK(H.), Le Destin de Iouri Voronine,Paris,Éd. de Fallois,2005. 
En dépit de la richesse qui l'entoure,Iouri vit dans le malheur.Il fait un portrait qui rend son fils antipathique et laisse prévoir le déclenchement des conflits entre les personnages.

Joe était un enfant"menteur"1 et démoniaque ${ }^{2}$.Adolescent,il est devenu perfide et agressif.Iouri nous révèle qu' "À seize ans, il est parti dans une maison de redressement pour trois ans.Vol et viol ${ }^{\prime \prime}$.Après sa libération,il n'est plus retourné chez lui ${ }^{4}$.Il est parti pour Los Angeles.Là,il Angeles.Là,il a fait peau neuve.Il a renié ses origines russes et prétendu être le descendant de la grande famille de Lincoln.

Connu sous le nom de "Joe Carson Lincoln" ,Joe devient un des puissants de la société américaine.Il fait de l'argent son idole: ami."

"(...), il appelle le dollar "mon Benjamin Franklin",ne connaît que cet

Ce matérialisme aveugle Joe.Il l'emplit d'égoïsme,d'insensibilité et le rend"(...)incapable d'aimer ${ }^{\prime \prime 7}$.Joe vit donc en incommunicabilité avec sa femme Mary:"(...), il la regarde à peine,lui parle peu ${ }^{\prime \prime}$.

Il n'arrive également pas à interagir avec son père qui lui représente "un faire-valoir" ${ }^{\prime \prime}$.En effet,Joe a trompé ${ }^{10}$ son père et l'a obligé à renoncer renoncer à son nom et à ses origines russes.Désormais,Iouri s'appelle"Erle carson Lincoln"11.

1)JELINEK(H.),Op.Cit,p.16.

2)"(...)il avait le diable dans la peau"dit-Iouri. Loc.Cit.

3) Loc.Cit.

4)Joe se contentait d'envoyer des cartes postales à ses parents.

5)Le nom russe de Joe était "Miroslav"La Paix glorieuse".

JELINEK(H.),Op.Cit,p.13.

6)ibid,pp.13-14.

7)ibid,p.112.

8)ibid,p.24.

9)http://www.evene.fr/livres/livre/henriette-jelinek-le-destin-de-iouri-voronine-16235.php consulté le 17/03/2009.

10)Joe a dit à son père qu'il allait signer les papiers de son départ de Chicago.Mais par ces papires,Iouri a été dépossédé de son nom et de ses origines russes.

11) JELINEK(H.),Op.Cit,p.17. 
Déraciné,Iouri est tourmenté par ce nom qui "(...) est comme une épine plantée droit dans (s)on ventre ${ }^{\prime \prime 1}$.Non seulement Joe a arraché le cœur de son père, mais encore il lui a ôté le désir de passer le reste de sa vie dans son pays natal ${ }^{2}$.

Pire encore,chez Joe,Iouri n'a trouvé aucune consolation.Il a été pris dans les engrenages de la vie mondaine avec ses exigences et ses interdits.Il n'est parvenu ni à renouer avec son fils autoritaire ni à communiquer avec sa belle-fille. Chacun évite l'autre et se replie sur soi-même: " Nous vivons presque dans le silence ${ }^{\prime 3}$, se plaint Iouri.

C'est la froideur qui règne dans la grande villa de Joe où tout respire l'hostilité et l'animosité:

"Ici,on passe son temps à être un ennemi pour l'autre(...)" ${ }^{\prime \prime 4}$ dit Mary à son beau-père.

De la sorte,nous allons essayer de montrer comment Jelinek a souligné le désaccord des interactants à travers l'étude de l'épisode polémique.

Mais quel est le sens de l'épisode ${ }^{5}$ ?

L'épisode"consiste en un ou plusieurs échanges poursuivant un objectif pragmatique commun et produits par un groupe constant d'interlocuteurs sur un thème donné" ${ }^{\prime \prime}$

Il y a deux types d'épisodes:phatiques et transactionnels.Les épisodes phatiques se composent des échanges rituels d'ouverture et de clôture souvent présupposés par le lecteur.Quant aux épisodes transactionnels,ils renvoient au corps même de l'interaction.Ils peuvent être de nature didactique,dialectique et polémique.L'épisode polémique se caractérise ${ }^{7}$

1)JELINEK(H.),Op.Cit,p.78.

2)Iouri dit:"Ce que je veux réellement,c'est retourner chez moi,en Russie,dans mon village(...)"

Loc, Cit.

3) ibid,p.24.

4) ibid,pp.122-123.

5)Le terme de l'épisode correspond à celui de "La séquence".

KERBRAT-ORECCHIONI(C.), Les interactions verbales.Approche interactionnelle et structure des conversations,TI,Paris,Éd.Armand Colin/Masson ,1998 (Troisième édition), p.213.

6)DURRER(S.),Le dialogue dans le roman,Paris,Éd.Nathan Université,1999,p.79.

7)Pour les autres caractéristiques des épisodes polémiques, voir ibid,pp.87-88. 
par l'affrontement des interlocuteurs et la divergence de leurs croyances qui sous-tendent l'enchaînement des différentes répliques ${ }^{1}$.

Or,tout épisode n'est pas une simple succession de répliques,mais une unité complexe relevant des règles d'organisation structurale.Nous allons donc nous évertuer à mettre en relief la dynamique fonctionnelle de ces règles à travers l'étude du couple complémentaire la cohésion et la cohérence discursives.

La cohésion correspond à "L'ensemble des moyens linguistiques qui assurent les liens intra- et interphrastiques permettant à un énoncé oral ou écrit d'apparaître comme un texte."

"L'endophore ${ }^{\prime 3}$,"Les parenthésages" ${ }^{\prime \prime 4}$, la progression thématique et l'isotopie représentent les principaux moyens qui permettent de constituer un fil conducteur sur le plan énonciatif et informatif.Ce qui favorise l'articulation des répliques et garantit,par la suite,l'intelligibilité discursive.

La cohésion mène à la cohérence dans la mesure où:

"Les marqueurs de cohésion ne sont que des indices d'une cohérence à construire par un travail interprétatifils ne sont là que pour faciliter ce travail. ${ }^{15}$

L'étude de la cohérence vise à cristalliser le"macro-acte de langage ${ }^{\prime \prime}$ de l'épisode conformément à sa nature.

1)"(...),sur le plan empirique, il est fréquent que les répliques correspondent à des interventions."

DURRER(S.),Op.Cit,p.74.

2)CHARAUDEAU(P.),MAINGUENEAU(D.),Dictionnaire d'analyse du discours , Paris , Éd.Du Seuil,2002,p.99.

3)"Le terme d'endophore fournit un hyperonyme aux expressions anaphore et cataphore.Pour cette raison,on parlera d'endophore anaphorique (...),et d'endophore cataphorique(...)" ibid,p.221.

L'anaphore et la cataphore sont basées sur l'idée de la reprise.Tandis qu'avec l'anaphore,le terme repris , l'antécédent ou l'anaphorisé, précède le terme qui reprend,l'anaphorique ou l'anaphorisant,avec la cataphore,le terme qui reprend,le cataphorique ou le cataphorisant,précède le terme repris,le cataphorisé.

4)"Avec les parenthésages,il s'agit d'étudier des ensembles de propositions reliées et hiérarchisées par des connecteurs(...) ou des organisateurs textuels(...)"

ADAM(J.-M),Les textes:types et prototypes,Paris,Éditions Nathan,1992(Troisième édition revue et corigée),p.27.

5) CHARAUDEAU(P.),MAINGUENEAU(D.),Op.Cit,p.100.

6)ibid,p.359. 
La cohésion et la cohérence seront soulignées à travers la mise en parallèle de deux exemples de l'épisode polémique ${ }^{1}$. Dans le premier épisode,Joe s'oppose à son père. Privé de la bonne compagnie et de l'amour,Iouri se sent emprisonné dans la villa de Joe:"C'est le bagne(...)" ${ }^{\prime 2}$ se plaint-il.Il est en proie à une solitude et d'une angoisse affreuses:"Je suis trop seul,trop seul." ${ }^{\prime 3}$ Il décide ,par conséquent,de s'évader de la villa et d'aller vivre avec John Ford":"Moi,je vivrais bien avec John Ford,il est gai,j'ai besoin qu'on me fasse rire. ${ }^{15}$ Mais Joe retrouve son père et lui reproche son attitude.

Dans le deuxième épisode,Joe se dispute également avec Mary.Son beau-père qui est "un des plus grands chercheurs de ce pays(l'Amérique $)^{\prime \prime 6}$ a refusé de l'aider à faire sortir $\mathrm{Neal}^{7}$, "son homme $d^{\prime}$ affaires ${ }^{\prime \prime}$, de la prison.

La cohésion va être étudiée sur le niveau inter/intra-répliques.

\section{**La cohésion inter-répliques:}

*L'endophore anaphorique:

L'endophore anaphorique correspond à la version standard de la reprise référentielle en ce sens que l'antécédent:"(..)est donné par le contexte linguistique. ${ }^{\prime \prime}$

Comme la reprise de l'antécédent se fait par plusieurs moyens,l'anaphore acquiert différentes formes:

\section{**'L'anaphore pronominale:}

Les noms personnels "je" et "moi" dans les 4ème et 11ème répliques de l'épisode $\mathrm{J} / \mathrm{I}$ ainsi que le pronom "me" complément des verbes "écouter,dire et trahir" dans les 7ème,13ème et 14ème répliques réfèrent au nom propre "Joe" dans la troisième réplique.Ce nom repris dans la 15ème réplique est également l'anaphorisé du pronom

1)Nous allons désigner le premier épisode par l'épisode $J(J o e) / I(I o u r i)$, et le deuxième par l'épisode $\mathrm{J}(\mathrm{Joe}) / \mathrm{M}($ Mary).Pour les deux épisodes, Voir Annexes pp.35-37.

2)JELINEK(H.),Op.Cit,p.72.

3)ibid,p.106.

4) Iouri a connu John Ford pendant une de ses promenades dans les collines d'Hollywood dans une limousine avec chauffeur.

5) JELINEK(H.),Op.Cit,p.73.

6) ibid,p.23.

7)Neal a été relâché:il"(...) a été pris pour un autre qui a tué sa maîtresse.L'assassin avait la même Continental grise et Neal ressemble au portrait-robot du meurtrier." ibid,p.34.

8) Loc.Cit.

9)KLEIBER(G.),Anaphores et pronoms,Belgique,Éd.Duculot,1994,p.57. 
"me"complément du verbe "aimer" dans la 16ème réplique,du "moi" et du "je" dans la 18ème réplique,du nom personnel "tu" dans la 19ème réplique et du déterminant possessif "mon"dans la 20ème réplique.

Au cours de l'épisode J/M le nom propre "Joe "constitue également le noyau d'"une chaîne anaphorique" assez prolifique.Dans le commentaire qui annonce l'épisode et sert à situer les interlocuteurs par rapport à leur discours,"Joe" est l'antécédent du nom personnel"toi" dans la 3ème réplique.Ce nom réapparaît dans le discours attributif des 4ème et 7ème répliques et remplit la même fonction référentielle auprès des noms personnels "tu"dans les 5ème,9ème,12ème répliques,"je" dans la 7ème réplique ,et du possessif "mon" dans la 10ème réplique.

En effet,les différentes occurrences de ce nom dans les deux épisodes marquent l'ampleur que gagne le rôle interlocutif de Joe et accentuent,par le fait même,le trait polémique de son apport discursif.

Parallèlement,le nom propre "Mary" déclenche un mouvement de reprises pronominales variées.Ce nom par lequel le narrateur débute le commentaire de l'épisode $\mathrm{J} / \mathrm{M}$ remplit le déterminant "ton"dans la première réplique et le pronom "te"complément du verbe "marier" dans la 2ème réplique.Il parcourt le discours attributif des 5ème et 9ème répliques et fait office de l'antécédent de "ton"dans les 7ème et 8ème répliques,du pronom "me" complément du verbe "ennuyer"dans la 9ème réplique et du "mon" dans la 12ème réplique.

Le nom propre ,dont les anaphorisants ont des formes diverses, fait écho au "substitut anaphorique" "il" qui peut avoir de multiples anaphorisés.Dans la 13ème réplique de l'épisode J/I,"il" résonne avec "lui" complément des verbes "raconter" et "dire" dans les 9ème et 11ème répliques et renvoie au "paradigme désignationnel"3 "un tueur de rats"dans la 6ème réplique.Ce substitut sillonne les 2ème,3ème et 4ème répliques de l'épisode $\mathrm{J} / \mathrm{M}$ et anaphorise le syntagme nominal"ton père" dans la première réplique.

En dépit de leur diversité,les anaphoriques pronominaux ont"(...) un effet de mise en saillance du syntagme anaphorisé. ${ }^{\prime 4}$

1)TISSET(C.),Analyse linguistique de la narration,Paris,Éd.Sedes,2000,p.138.

2)Les substituts anaphoriques sont des "pronoms traditionnels de troisième personne, déterminants possessifs, certains groupes nominaux définis,correspondent à la classe des anaphores ou chaînes de référence(...)".

ADAM(J.-M),Linguistique textuelle.Des genres de discours aux textes,Paris,Éditions Nathan,1999,p.46.

3)Les paradigmes désignationnels correspondent aux"(...)diverses expressions qui sont autant de substituts linguistiques du nom."

TISSET(C.),Op.Cit,p.28.

4) ibid,p.33. 
Ils contribuent,par la suite, à assurer l'alternance syntactico-sémantique des répliques.Ils constituent également un phénomène de reprise totalement $^{1}$ coréférentielle ${ }^{2}$ le pronom possessif féminin singulier"la mienne"dans la 6ème réplique fait référence à "une autre expression" dans la 5 ème réplique de l'épisode $\mathrm{J} / \mathrm{M}$.

Les anaphoriques pronominaux sont donc des

"(...) pronoms représentants(...),qui varient en genre et en nombre,(...),des véritables pro-noms,c'est -à- dire des éléments qui reprennent une autre unité du cotexte. ${ }^{13}$.

Ceci est d'autant plus mis en relief à travers le pronom invariable neutre "ça" dans la 4ème réplique.Par ce démonstratif à valeur "résomptive $e^{\prime 4}$,Joe renvoie à la totalité énonciative de la réplique précédente de sa femme. Le pronom"ce" dans la 13ème réplique permet également à Joe de récapituler le contenu de la réplique de son père.Ce qui mène à la progression de l'enchaînement discursif.

\section{**L'anaphore nominale:}

"Une anaphore nominale ${ }^{\prime 5}$ infidèle fondée sur la relation de synonymie apparaît dans la 6ème réplique de l'épisode $\mathrm{J} / \mathrm{I}$ à travers le paradigme désignationnel "un tueur de rats" qui renvoie au nom propre "John Ford" dans la 5ème réplique.Ce paradigme a pour fonction "(...) de thématiser le rôle social ou familial du personnage."

1)L'endophore pronominale peut être totale quand les anaphoriques pronominaux renvoient à l'entité référentielle de l'antécédent,ou partielle quand,au contraire,ils ne reprennent que partiellement l'antécédent.

2)Il y a coréférence lorsque certains mots ou expressions désignent le même référent.

3)MAINGUENEAU(D.),Analyser les textes de communication,Paris,Éd.Nathan,2000,p.175.

4)"Une anaphore ou une cataphore résomptive ou conceptuelle condensent un fragement de niveau au moins égal à la phrase."

ibid,p.173.

Mais "Une anaphore ou une cataphore dite "segmentale" reprend une unité de niveau inférieur à la phrase"

Loc.Cit.

5)Les anaphores nominales "sont dites fidèles lorsqu'elles ne sont que la reprise du même mot avec un changement de déterminant(...)"

TISSET(C.),Op.Cit,pp.32-33.

Mais"Elles sont dites infidèles lorsque la reprise se fait par des éléments lexicaux. "Parmi ces éléments figurent la relation de synonymie,d'antynomie et de métaphore. ibid,p.33.

6) ibid,p.28. 
La même relation s'établit dans l'épisode $\mathrm{J} / \mathrm{M}$ entre les paradigmes"ton professeur de biologie"dans la 8ème réplique et "ton père"dans la réplique précédente.

La synonymie sous-tend également la coréférence entre "les axiologiques négatifs" "espèce de putain,un bol de merde,menteuse,dégueulasse",dans les 10ème,11ème ,13ème et 15ème répliques et le nom de "Mary" dans le discours attributif de la 9ème réplique.Ces insultes aussi diverses que successives marquent non seulement la discordance du couple Joe/Mary mais encore une escalade dans la dynamique polémique qui conditionne le parcours discursif.

L'anaphore nominale infidèle peut également être de type conceptuel: le syntagme nominal"une autre expression" dans la 5ème réplique réfère au contenu significatif de la 4ème réplique.

\section{**L'endophore cataphorique:}

Parallèlement à l'anaphore,la cataphore a plusieurs types dont:

\section{* La cataphore pronominale:}

La personne "je" dans la première et la 2ème répliques de l'épisode $\mathrm{J} / \mathrm{I}$ est le cataphorisant du nom propre "Joe" dans la 3ème réplique.

En effet,avancer le terme qui reprend avant le terme repris suscite l'attente du lecteur et éveille sa curiosité.

\section{**l'exophore:}

L'exophore s'oppose à l'endophore.Elle renvoie à la version pragmatique fondée sur le critère de la saillance du référent dans la mémoire discursive:

"Dans un tel cadre,(...),l'anaphore devient un processus qui indique une référence à un référent déjà connu par l'interlocuteur,c'està-dire un référent présent ou déjà manifeste dans la mémoire immédiate, appelée (...)mémoire discursive(...) ou encore focus (...)" ${ }^{2}$

Plus précisément,dans l'exophore,le couple anaphorisé/cataphorisé est absent du contexte linguistique des répliques.Pour le récupérer,il ne suffit pas de s'appuyer sur "le référent saillant ${ }^{\prime 3}{ }^{3}$,mais il faut également prendre en considération le contenu de la phrase- hôte:

\footnotetext{
1)Les axiologiques sont" des termes péjoratifs(...),mélioratifs(laudatifs,valorisants)." KERBRAT-ORECCHIONI(C.),L'énonciation de la subjectivité dans le langage,Paris, Éd.Armand Colin/Masson,1997(Troisième édition),p.73.

Les interlocuteurs ont eu recours aux axiologiques négatifs"dont l'injure constitue un emploi discursif particulier." ibid,p.74.

2)KLEIBER(G.),Op.Cit,p.25.

3)Le référent saillant correspond à :"(...) une entité suffisamment pertinente cognitivement pour apparaître comme le référent d'une prédication."

ibid,p.83.
} 
"Il faut,(...),que ce qui est dit,dans la phrase-hôte du référent choisi pour le pronom soit pertinent.S'il n'en va pas ainsi:le référent recruté est éliminé et c'est un autre candidat qui se trouve appelé."I

Le référent des noms personnels "je"dans la 12ème,la 15ème répliques,"tu"dans la 6ème,7ème,9ème,11ème,14ème,16ème,18ème et 20ème répliques et du pronom tonique"toi"dans la première et la 4ème répliques et de "te"complément du verbe "dire" dans la 2ème réplique n'est pas fourni par le contexte de l'épisode $\mathrm{J} / \mathrm{I}$.Mais conformément à la saillance référentielle et au contenu des phrases-hôtes,c'est Iouri qui représente le bon référent.

De la sorte,l'exophore fait corps avec l'endophore pour promouvoir "la concaténation" ${ }^{2}$ des répliques:

"Anaphore et cataphore instaurent une relation de coréférence entre le terme source et le terme repris ou annoncé. Cette relation assure la progression de l'information et confère une cohésion au texte ${ }^{13}$

La continuité discursive s'appuie sur cette progression de l'information voire sur la progression thématique qui consiste à ce que:

$$
\text { "(...)tout texte est fait d'équilibre entre l'apport }
$$

d'information( appelé rhème)et les éléments connus (appelés thèmes) ("4 $^{\prime 4}$

La succession des thèmes et des rhèmes aboutit donc à la constitution d'une entité discursive et narrative intelligible.

Il y a une progression "à thème constant" ${ }^{\prime 5}$ dans les 2ème et 3ème répliques de l'épisode $\mathrm{J} / \mathrm{M}$ à travers la répétition du même thème"il".D'une part,l'occurrence d'un seul thème enrichi par des rhèmes différents,"ne crânait pas...marier, ne voulait pas ...toi" ${ }^{\prime \prime}$, permet de jeter la lumière sur les multiples facettes de la personnalité du père de Mary.D'autre part,elle donne du relief à la dimension agonale que gagne le rôle de ce personnage dans l'épisode.

La progression thématique ,qui"(...) instaure une continuité dans la trame narrative" ${ }^{\prime 7}$, s'accorde avec les parenthésages qui"(...) marquent l'articulation transphrastique et soulignent la progression générale de la pensée. ${ }^{\prime 8}$

1)KLEIBER(G.),Op.Cit,p.108.

2)"La concaténation est un phénomène d'enchaînement qui assure la liaison des répliques entre elles sur les plans thématique,syntaxique et pragmatique."

CALAS(F.),CHARBONNEAU(D.-R),Méthode du commentaire stylistique,Paris, Éditions

Nathan,2000,p.207.

3)ibid,p.17.

4)TISSET(C.),Op.Cit,p.36.

5) La progression peut aussi être linéaire ou en thèmes dérivés. ibid,p.38.

6)JELINEK(H.),Op.Cit,p.38.

7)CALAS(F.),CHARBONNEAU(D.-R), Op.Cit,p.16.

8)Loc.Cit. 
Pour s'imposer dans le discours et garder leur position de force,les interlocuteurs ont recours à plusieurs connecteurs dont le plus retentissant est le "mais"1

**Mais:

Dans la 8ème réplique Iouri ne répond pas à la question de son fils.Il enchaîne par "mais"qui dénote son opposition au discours antérieur de Joe.En effet, Iouri a tenté de s'expliquer pour apaiser la colère de son fils qui est en train de le harceler de ses répliques.Cependant,sa tentative n'a pas abouti à cause de l'interruption négative ${ }^{2}$ de son tour de parole.

Joe a également recours à "mais" dans la 18 ème réplique pour rejeter la question de son père,"(...)pourquoi on en meurt tous?" ${ }^{\prime 3}$, et lui déclarer son désaccord.À vrai dire,cette opposition des répliques de Joe/Iouri marque la discordance de leurs points de vue.

Outre l'opposition,"mais"a une fonction phatique"d'établissement-prise de parole ${ }^{\prime \prime}$ dans la 7 ème réplique de l'épisode J/M.Ce "mais" contribue également à "articuler les morceaux discursifs a priori hétérogènes" ${ }^{15}$ tout tout en marquant une phase discursive.Joe signale le passage de son discours belliqueux aux menaces de mort proférées contre son beaupère"Mais je peux...jamais"

Parallèlement à"mais",les interlocuteurs ont recours à "donc" et à "si". **Donc:

Donc" représente un "des connecteurs de "consécution"" qui ont

1)À la valeur d'opposition du "mais",Adam(J.-M)ajoute cinq autres fonctionnements: mais $<1>$ de renforcement-renchérissement, mais $<2>$ réfutatif,mais $<3>$ phatique et/ou de démarcation de segments textuels,mais $<4>$ concessif,et mais $<5>$ argumentatif.

$\mathrm{ADAM}(\mathrm{J} .-\mathrm{M})$, Éléments de linguistique textuelle.Théorie et pratique de l'analyse textuelle,Bruxelles-liège, Éd.Mardaga,1990 Deuxième édition,pp.192-211.

2)L'interruption représente un des ratés des tours de parole qui entrave l'enchaînement discursif.Néanmoins,il y a des "interruptions à fonction positive d'entraide."

KERBRAT-ORECCHIONI(C.),Les interactions verbales.Approche interactionnelle et structure des conversations, TI,p. 177.

3) JELINEK(H.),Op.Cit,p.83.

4) $\mathrm{ADAM}(\mathrm{J} . \mathrm{M})$,Éléments de linguistique textuelle.Théorie et pratique de l'analyse textuelle,p.200.

5)ibid,p.203.

6) JELINEK(H.),Op.Cit,p.39.

7)MAINGUENEAU(D.),Nouvelles tendances en analyse du discours , Paris , Éd. Hachette , 1987,p.128. 
"(...)une activité de déduction que l'énonciateur prétend effectuer personnellement."

Dans "P donc Q", Q est considéré comme la conclusion de P.

Ainsi ,dans la 17 ème réplique,Iouri se sert-il du "donc" pour répondre à son fils qui s'indigne"Tu m'aimes ...m'aimes?"

Le système déductif ${ }^{3}$ de "donc" peut être illustré de la manière suivante:

a)Joe demande à son père comment il l'aime.

b)Joe ne parvient pas à sentir que son père l'aime.

c)Si Joe ne parvient pas à sentir que son père l'aime et lui demande comment il l'aime,alors,il ne comprend pas que son père s'est évadé parce qu'il ne pouvait pas supporter sa richesse qui l'écoeure.

d)Joe ne comprend pas que c'est la richesse qui écoeure son père (d) est la conclusion qui résulte de (a),(b) et(c).(a) équivaut à l'énoncé de Joe et (b) à la prémisse impliquée ${ }^{4}$.La relation entre (a) et (b) est mise en exergue par (c) qui engendre (d).

\section{**Si P,Q:}

Le si hypothétique indique que la réalisation de $\mathrm{Q}$ est entièrement dépendante de celle de P.Face à Joe qui attaque farouchement son père,Mary s'emporte:

"-Si mon père est ce que tu dis, le tien est un vieux vicieux qui aime trop mes seins et mon derrière..$^{15}$

Si suivi du présent de l'indicatif dénote que l'interlocuteur"(...)pose la condition comme remplie au moment où il parle. ${ }^{1{ }^{6}}$

Mary a donc employé le présent "est" pour prouver que son beau-père mérite d'être l'objet de son accusation infamante.

1)ANSCOMBRE(J.-M),DUCROT(O.),L'argumentation dans la langue, Bruxelles ,

Éd.Mardaga , 1983,p.87.

2)JELINEK(H.),Op.Cit,p.83.

3)Nous allons nous référer à MOESCHLER(J.),Modélisation du dialogue.Représentation de l'inférence argumentative,Paris,Éd.Hermès, 1989,p.180.

4)Les prémisses correspondent aux hypothèses contextuelles qui "(...)sont accessibles à partir de diverses origines:l'environnement physique de l'individu,la mémoire à long terme(...),la mémoire à court terme(...)ou encore la mémoire du mécanisme déductif contenant les hypothèses liées aux énoncés traités précédemment."

ID,Théorie pragmatique et pragmatique conversationnelle,Paris,Éd.Armand Colin, 1996,p.212.

5) JELINEK(H.),Op.Cit,p.39.

6)LÉVY(M.),PERSEC(S.),Grammaire du français.Approche énonciative, Paris,Éditions OPHRYS,2000,p.220. 


\section{**La cohésion intra-répliques:}

Sur le plan intra-répliques,les connecteurs font également office d'un élément de prime importance.

**Mais argumentatif:

Ce "mais" est à l'intérieur d'une réplique.Il relie deux propositions anti-orientées $\mathrm{P} / \mathrm{Q}$ et est décrit selon le schéma standard du carré argumentatif ${ }^{1}$.

Dans la 2ème réplique de l'épisode J/I ,Joe jette la pierre au comportement de son père:

"-Les Russes aiment la comédie,faire beaucoup de théâtre mais, avec moi, ऽa ne marche pas."

La première proposition $\mathrm{P}, "$ Les Russes...théâtre."pourrait avoir la conclusion "R"="Ton comportement est légitimé par le fait que tu sois russe".Cette conclusion est infirmée par la deuxième proposition $\mathrm{Q}$,"avec...pas",qui pourrait avoir la conclusion "non-R"="Je n'approuve pas ton comportement."

Iouri essaie de se justifier dans la 17ème réplique.Il explique à son fils qu'il se sent étouffé par sa richesse:

"Je l'accepte ta richesse mais pourquoi on en meurt tous?"13

La première proposition $\mathrm{P}, " J e$...richesse",pourrait avoir la conclusion "R"=" Ta richesse ne me cause aucun problème."

La situation est renversée par la deuxième proposition Q, "pourquoi...tous?",qui pourrait mener à une conclusion diamétralement opposée"non-R":"Ta richesse me pèse lourdement au point de me tuer". L'effet dominant de cette conclusion est relatée par la valeur indéfinie et générale du "on"et de "tous".

De même,dans la première réplique de l'épisode $\mathrm{J} / \mathrm{M}$,Joe condamne l'attitude de son beau-père qui l'a abandonné et refusé de sauver Neal de la prison:

"-(...)il me prend pour un pauvre mec,mais qu'il fasse attention,ton père! "'tit-il à Mary

La conclusion de la première proposition $\mathrm{P}$,"il me...mec",pourrait être"R"="Je suis considéré par ton père comme une personne impuissante".Joe enchaîne par "mais" qui change radicalement la situation et lui permet de lancer une menace à sa femme.Q,"qu'il...père",pourrait avoir la conclusion "non-R"="Que ton père comprenne que je suis une personne puissante.

1)Voir MAINGUENEAU(D.),Nouvelles tendances en analyse du discours,p.122.

2)JELINEK(H.),Op.Cit,p.82.

3)ibid,p.83.

4)ibid,p.38. 
À Mary qui essaye de défendre son père,Joe répond par une nouvelle opposition dans la 4ème réplique:

"-On dit ऽa,mais il frétillait le salaud."

La proposition $\mathrm{P}, "$ On dit $\varsigma a^{\prime \prime}$,illustre bien le topos ${ }^{2}$ convoqué:"Plus on voit un nouveau riche se marier avec une femme issue d'une grande famille,plus on répète les mêmes paroles".Ce topos introduit la conclusion $\mathrm{R}=" \mathrm{Tu}$ as raison"." $\mathrm{R} "$ est bouleversée par $\mathrm{Q}$,"il...le salaud",qui pourrait donner le jour à la conclusion opposée"non-R"="Tu as tort"

Le "mais argumentatif" résonne avec le "mais" de la 12ème réplique de l'épisode J/M.Ce "mais" marque l'opposition de Mary au regard de Joe dont la valeur implicite est la désapprobation.Cette opposition est mise en relief par le "oui" et l'injonction négative "ne me regarde pas comme $\varsigma a !^{\prime \prime 3}$ qui peut être paraphrasée par "Tu ne dois pas me regarder ainsi parce que j'ai toute raison de dire ce que je dis".Pour étayer son argument, Mary fait allusion à l'attitude antérieure de son beau-père"Viens...lèvres."

\section{**Mais phatique:}

Ce mais parcourt les 14ème et 16ème répliques de l'épisode J/I.Dans la 14ème réplique,il articule deux propositions:

"tu cours le risque de nous amener quelques criminels,,mais n'est-ce pas,qu'est-ce que ça fait?"" .

La première proposition va dans le sens du renforcement de la deuxième.Joe déclare à son père l'effet dangereux de son attitude.Il veut lui faire reconnaître ce danger à travers une interrogation négative à valeur affirmative.Joe enchaîne:"Tu me détestes à ce point,mais dis-le" $e^{\prime 5}$. Ici "mais" "marque une opposition du locuteur au destinataire,motivée par un comportement immédiatement précédent de ce dernier"

Cette opposition est d'autant plus accentuée,au cours de la 16ème réplique par l'itération de la même proposition mais avec un changement

\footnotetext{
1)JELINEK(H.),Op.Cit,p .38.

2)Dans la théorie de "l'argumentation dans la langue": "les topoï sont des principes généraux,communs,(...), mettant en relation graduelle des propriétés(prédicats ou échelles)elles-mêmes graduelles."

CHARAUDEAU(P.),MAINGUENEAU(D.),Op.Cit,p.579.

3) JELINEK(H.),Op.Cit,p .39.

4)ibid,p.83.

5)Loc.Cit.

6)DUCROT(O.),Les mots du discours,Paris,Les Éditions de Minuit,1980,112.
} 
de voix:de l'affirmation"Tu m'aimes, tu dis que tu m'aimes" "1'interlocuteur passe à l'interrogation:"mais comment tu m'aimes?"2 qui assure à la fois son indignation et le rejet de l'acte de parole précédent de son père.

***Mais réfutatif:

Ce "mais" apparaît dans la 19ème réplique de l'épisode J/I :

"-Tu ne seras jamais un prince mais un miteux,un nouveau riche...admirer ${ }^{\prime \prime 3}$

L'interprétation réfutative ${ }^{4}$ de ce "mais"peut être décrite comme ce qui suit:on admet le fait que grâce à ta fortune tu seras un prince \{proposition $\mathrm{P}$ \}, or il n'en est rien (cela est faux)\{proposition NON-P\},mais je peux,en revanche, vous dire(=cela est VRAI)que $\{$ propositionQ $\}$.

**Mais concessif:

Le "mais concessif" " est révélat(eur) des présuppositions du locuteur. ${ }^{\prime 5}$ Après avoir lu le rapport sur John Ford à son père,Joe dit :

"-Bref mais clair."

La conclusion impliquée par l'épithète"Bref" va dans le sens de NON-Q.La présupposition est la suivante:"Un rapport bref n'est généralement pas clair."Mais" renverse cette présupposition en introduisant "Q".La relation de concession qu'il établit peut être illustrée à travers le schéma suivant:

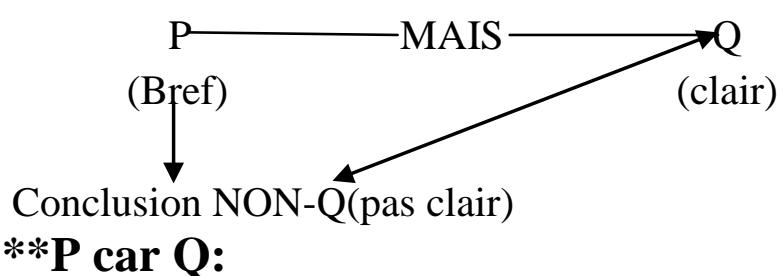

Dans P car Q,il y a une succession de deux actes d'énonciation:

"(...) le second est présenté comme destiné à légitimer le premier,cette légitimation peut porter sur le droit d'énoncer(...) ou,le plus souvent,sur le fait de présenter $Q$ comme une raison de croire $P$ vrai. ${ }^{7}$

1)JELINEK(H.),Op.Cit,p.83.

2)Loc.Cit.

3)JELINEK(H.),Op.Cit,p.84.

4)Nous suivrons l'exemple analytique du"mais réfutatif" proposé par $\operatorname{ADAM}(\mathrm{J} .-\mathrm{M})$, Eléments de linguistique textuelle.Théorie et pratique de l'analyse textuelle,p.196.

5)ibid,p.204.

6)JELINEK(H.),Op.Cit,p.83

7) MAINGUENEAU(D.),Nouvelles tendances en analyse du discours,p.126. 
Joe menace d'écraser son beau-père.Il affirme à sa femme que sa menace va lui coûter cher:

"-(...) tu attendras longtemps pour le retrouver,car tu ne le retrouveras jamais." ${ }^{1}$

Joe se justifie d'avoir dit P,"tu...retrouver",par Q"tu...jamais". À vrai dire,Mary risque de perdre à jamais son père au cas où son mari mettrait sa menace à exécution.

\section{**Si P,Q:}

À son père qui dit que la richesse le fait mourir,Joe rétorque:

"-Peut être que si tu en avais fais autant,je pourrais me reposer,je ne mourrais pas,comme tu dis." ${ }^{2}$

Au cours de cet exemple, "si" établit une relation hypothétique irréelle entre $\mathrm{P}, " t u \ldots a u t a n t "$, et $\mathrm{Q}, " j e . . . p a s "$.Le plus que parfait ainsi que le conditionnel signalent que ces énoncés se déroulent dans un monde imaginaire puisqu'ils ne sont pas réalisés et ne se réaliseront plus.Ceci est souligné par le modélisateur ${ }^{3}$ épistémique "peut-être"qui exprime l'incertitude.En effet,Joe reproche à son père de ne pas s'être acquitté de ses obligations envers lui:Iouri aurait dû s'efforcer d'offrir une vie aisée à son fils pour lui épargner beaucoup de difficultés.

À l'instar des connecteurs, les marqueurs d'intégration linéaire servent parfaitement la jointure des répliques.Ils ont pour fonction"(...)de structurer la linéarité du texte,de l'organiser en une succession de fragments complémentaires qui facilitent le traitement interprétatif. ${ }^{15}$ Il y a des marqueurs d'intégration linéaire d'ouverture,de relais et de clôture."Puis",marqueur de relais,articule la succession linéaire et chronologique $^{6}$ des informations données sur John Ford dans la 5ème réplique de l'épisode $\mathrm{J} / \mathrm{I}$ :

1)JELINEK(H.),Op.Cit,p.39.

2)ibid,p.84.

3)"Les modélisateurs"renvoient aux"adverbes subjectifs"

TISSET(C.),Op.Cit,p.180.

4)"La modalité épistémique (...) évolue le certain et l'incertain,à partir de l'expérience du locuteur(...)"

CALAS(F.),CHARBONNEAU(D.-R),Op.Cit,p.49.

5)MAINGUENEAU(D.),Éléments de linguistique pour le texte littéraire,Paris,Éd.BORDAS, 1990(Nouvelle édition),p.142.

6)"Puis" figure également parmi les organisateurs temporels qui"(...)fonctionnent comme des marqueurs d'intégration linéaire."

ADAM(J.-M), Éléments de linguistique textuelle.Théorie et pratique de l'analyse textuelle, p.161. 
"-John Ford,(...),établi à San Francisco,puis Las Vegas,puis Los Angeles." ${ }^{1}$

"Puis" fait écho à "et" qui signale la clôture de la 2ème réplique:

"Et je suis venu te le dire."

Ce "et de clôture ${ }^{\prime 3}$,équivalent d'un enfin, vient terminer le message que Joe a voulu adresser d'emblée à son père.

\section{**La progression thématique:}

Il y a une progression à thème constant dans la 14ème réplique de l'épisode J/I.La répétition du thème "tu" dans des énoncés à rhèmes variés dénote que Joe est en train d'accabler son père de critiques aussi acerbes que successives.

\section{**'L' endophore anaphorique: \\ *L'anaphore pronominale:}

Le substitut anaphorique "son" et le pronom "lui" dans la première réplique de l'épisode $\mathrm{J} / \mathrm{M}$ réfèrent à "ton père".Les trois substituts successifs,"il", sujets des verbes "être,blesser et prendre" anaphorisent également le paradigme désignationnel "le sinistre con".

Parallèlement,"il" dans la 19ème réplique de l'épisode J/I renvoie aux paradigmes"un miteux, un nouveau riche".Ce substitut résonne avec le pronom "te" complément du verbe"ennuyer" dans la 10ème réplique de l'épisode $\mathrm{J} / \mathrm{M}$, et avec le nom personnel "tu" dont l'antécédent est "espèce de putain".Aux"te" et"tu" répond le pronom"le" qui,complément du verbe "faire cimenter,retrouver,et retrouveras" dans la 7ème réplique anaphorise "ton père".Ce pronom apparaît également dans la 12ème réplique,"Viens le voir installé ${ }^{\prime \prime 4}$,et fait office de l'anaphorique d'un vieux vicieux".

"Le" peut aussi avoir une valeur résomptive.Complément du verbe "dire",dans les 2ème et 14ème répliques de l'épisode J/I, "le" renvoie à l'ensemble énonciatif précédent."Le" coïncide avec "ça" qui sous-tend une reprise énonciative récapitulative dans les 2ème et 17ème répliques de l'épisode J/I.Dans la 6ème replique "ça" introduit une reprise dévalorisante du paradigme"un tueur de rats".

1)JELINEK(H.),Op.Cit,p.82.

2)Loc.Cit.

3)TISSET(C.),Op.Cit,p.118.

4)JELINEK(H.),Op.Cit,p.39. 
Ici,"ça" "(...) opère un mouvement de déférenciation,de neutralisation(forcément disqualifiante) d'un référent à l'identité par cela même estompée."

\section{*L'anaphore nominale:}

Elle est de type infidèle.La reprise du nom propre"John Ford" dans la 5ème réplique de l'épisode $\mathrm{J} / \mathrm{I}$ se fait par un élément lexical synonyme"exterminateur".Le cas se répète dans la première réplique de l'épisode $\mathrm{J} / \mathrm{M}$ où les paradigmes "ce bol de merde et le sinsitre con" réfèrent au syntagme nominal "ton père" et "un homme présumé encore criminel" anaphorise le nom "Neal".Ces axiologiques négatifs ajoutent à la caractérisation des interlocuteurs et à la teneur psychologique de leur discours.

\section{**'L'exophore:}

Dans la première réplique de l'épisode $\mathrm{J} / \mathrm{I}$,"tu" fait référence à "toi" rempli en vertu du critère de la saillance référentielle par le nom de Iouri.

\section{**L'endophore cataphorique:}

\section{* La cataphore pronominale:}

Les liens cataphoriques intra-répliques reposent particulièrement sur les pronoms compléments des verbes.Dans la 14ème réplique de l'épisode J/I,"me" complément du verbe"trahir" cataphorise "ton fils".Dans la réplique suivante,"te" complément du verbe "aimer" est le cataphorisant de "Joe"."Le" complément du verbe "accepter" a pour cataphorisé "ta richesse" dans la 17ème réplique.Le même pronom, complément du verbe "briser", dans la 17ème réplique de l'épisode J/M est rempli par "ton père".

Le substitut anaphorique"il"sujet du verbe "faire attention" dans la première réplique réfère à "ton père" et à "le salaud" dans la 4ème réplique."Tu" dans la 19ème réplique de l'épisode J/I renvoie à une chaîne paradigmatique diverse:"un prince,un miteux et un nouveau riche".Les noms personnels,les substituts anaphoriques ainsi que les compléments contribuent donc, pour une grande part, à la jonction cataphorique discursive.

L'endophore/l'exophore,la progression thématique et les parenthésages s'accordent, sur les niveaux inter/intra-répliques, avec l'isotopie qui

"joue un rôle prépondérant dans l'organisation d'un texte et assure sa cohésion par les rapprochements qu'elle ménage entre les termes" ${ }^{\prime 2}$.

1)MAINGUENEAU(D.),Éléments de linguistique pour le texte littéraire,p.159.

2)CALAS(F.),CHARBONNEAU(D.-R),Op.Cit,p.38. 
L'imporatnce de l'isotopie s'explique par le fait qu'elle correspond à un

"(...),réseau fédérateur de termes comprenant un sème définitoire commun."

Plusieurs isotopies s'enchevêtrent tout le long des deux épisodes qui deviennent,par la suite, "polyisotop(ique)"2

\section{**L'isotopie de la parole:}

Cette isotopie se trouve à la base des interactions verbales.Elle repose sur le champ lexical de la parole argumentative mise en relief par les différentes modalités interrogatives et injonctives dans les deux épisodes:"tais-toi,dis-moi,ne me regarde pas,viens le voir".Elle est également illustrée par les verbes relevant de la parole:"dire,se taire,raconter,divulguer,répondre."

\section{**L'isotopie grammaticale:}

Elle est soulignée par les temps verbaux panchroniques"qui s'étend(ent) à l'ensemble des époques de la chronologie(passé,présent,futur). ${ }^{13}$

$\mathrm{Au}$ fur et à mesure, les interactants ont eu recours au présent qui actualisent les événements énonciatifs, "dors, t'encanailles , veux,aime,comprends,est,peux,entends..",à l'imparfait de la description, "crânait, frétillait" et au futur,"attendras,dormiras,seras."

\section{**L'isotopie de la relation à autrui:}

Elle unit plusieurs champs lexicaux:

\section{*Les relations antagonistes:}

La dimension agonale des relations interpersonnelles gagne de l'envergure grâce aux verbes qui parcourent:

-Le premier épisode:"trahir,ne pas respecter,détester,asservir, écraser, tromper,profiter de".

-Le deuxième épisode:"refuser son aide à, blesser,briser,faire cimenter". Le champ lexical des relations antagonistes est corroboré par celui de "la caractérisation de l'autre" fondé sur les insultes:

-Dans le premier épisode:"des menteurs,la racaille,criminels, un miteux,un nouveau riche."

-Dans le deuxième épisode:"un bol de merde,un pauvre mec,le salaud,espèce de putain,un vieux vicieux,menteuse,dégueulasse."

1)CALAS(F.),CHARBONNEAU(D.-R),Op.Cit,p.38.

2)Loc.Cit.

3)ibid,p.239. 
L'isotopie,l'endophore/l'exophore,la progression thématique et les parenthésages s'enchêvetrent pour donner naissance à la cohérence discursive.

\section{**La cohérence:}

La cohérence porte sur l'étude globale des composantes discursives:

"(...)sur l'analyse de l'implicite(...),sur l'orientation de l'argumentation vers un but(...),sur le respect du principe de coopération et de maximes de conversation(notamment, la maxime de pertinence)." ${ }^{1}$

C'est Joe qui ouvre les deux épisodes.Il occupe,par la suite,une position haute par rapport à Iouri et à Mary dans la mesure où: parole $^{\prime 2}$.

"la "maîtrise" interactionnelle est directement liée à la prise de

Il commence par attaquer son père ${ }^{3}$ à travers une injonction négative implicitée par un acte d'assertion:"(...)toi non plus, tu ne dormiras pas! "14

Cette dérivation allusive ${ }^{5}$ peut être explicitée de la façon suivante:

-Valeur assertive:primitive et principale.

-Valeur jussive:dérivée et secondaire (connotée).

Joe veut mettre son père en garde contre toute tentative d'évitement.La valeur polémique de son acte injonctif tient surtout à la transgression des règles de la politesse:Joe s'arroge le droit de demander des comptes à son père.De la sorte,il refuse de reconnaître l'autorité paternelle et se déclare quitte de ses devoirs d'obéissance et de respect.Ce qui marque l'inversion des rapports de force qui s'instaure, d'emblée,entre le père/le fils et régit leur interaction verbale.

1)SIOUFFI(G.),RAEMDONCK(D.V.),100 Fiches pour comprendre la linguistique , Paris, Éd.Bréal,Rosny,1999,p.113.

2)KERBRAT-ORECCHIONI(C.),Les interactions verbales,TII,Paris,Éd.Armand Colin , 1992,p.84.

3)C'est Neal qui est allé chercher Iouri du commissariat.De retour à la maison,Iouri n'a trouvé ni Joe ni Mary.Il consulte l'Encyclopedia Britannica pour lire la vie d'Abraham Lincolin.Mais, Iouri ,qui a peur de son fils, s'effraye en écoutant le bruit de sa voiture.Il se hâte d'aller se coucher et de cacher l'Encyclopedia sous les couvertures.

4) JELINEK(H.),Op.Cit,pp.82.

5)L'implicite intentionnel a deux formes:la dérivation allusive et le trope illocutoire lexicalisé. Avec la dérivation allusive ou le sous-entendu illocutoire,la valeur dérivée de l'énoncé est non-conventionnelle(n'a pas de marqueurs).Elle"(..)vient s'ajouter à la valeur primitive sans avoir toutefois la force de s'y substituer,et de servir à sa place de base pour l'enchaînement." KERBRAT-ORECCHIONI(C.),L'implicite,Paris, Éd.Armand Colin,1998(deuxième édition), p.76.

Par contre,en cas du trope, la valeur dérivée est conventionnelle(a des marqueurs de nature syntaxique,lexicale ou prosodique). 
Pour contraindre son père à se lever,Joe n'hésite pas à commettre une offense proxémique:"Il s'approche $d u$ lit"1. Il cingle son père d'une réplique impitoyable à valeur de reproche implicite:Joe reproche à son père d'avoir emboîté le pas à ses compatriotes russes qui misent sur la duperie.En effet,Iouri se trouve doublement blessé par cette réprimande.Non seulement son fils a condamné son attitude,mais encore a renié son pays natal auquel il s'attache par des liens inextricables.Joe a choisi de clore sa réplique par un avertissement,"Et je suis venu te le dire $^{\prime 2}$,qui résonne avec son reproche.

Joe s'insurge également contre sa femme.Il attaque avec frénésie son beau-père qui s'est abstenu à venir à son secours.Les injures,"bol de merde,le sinistre con",ainsi que le discours rapporté sous forme de modalités interrogatives, ${ }^{\prime L a}$ loi...criminel? ${ }^{\prime 3}$, révèlent la colère et l'indignation de Joe.Celui-ci ne tarde pas à accabler sa femme d'une menace implicite, "Qu'il fasse attention,ton père!", ,dont la violence est dénotée par le point d'exclamation qui produit un effet de martèlement.

Mais,Mary ne bronche pas.Elle adopte une attitude hautaine et ne réagit pas à l'intervention initiative de Joe.Cette "troncation" ${ }^{\prime 5}$ considérée comme "une offense grave "I6 $^{6}$ permet à Mary de damer le pion à son partenaire communicatif: elle s'élève au dessus de son énoncé et refuse, par le fait même, de s'engager dans le cadre interactionnel qu'il lui propose.Ce qui provoque Joe davantage.Ainsi enchaîne-t-il par une réplique agonale où il jette farouchement la pierre à son beau-père.L'adverbe "autant"sert bien la critique de Joe.Il est "le support signifiant" d'un "présupposé" ${ }^{\prime 8}$ qui renvoie

1)JELINEK(H.),Op.Cit,p.82.

2)Loc.Cit.

3) ibid,p.38.

4)Loc.Cit.

5)Il y a troncation quand"(...) une intervention à prétention initiative ne donne lieu à aucune réaction verbale ou non verbale:elle n'est tout simplement pas prise en compte par L2 (locuteur) ".

KERBRAT-ORECCHIONI(C.),Les interactions verbales.Approche interactionnelle et structure des conversations, TI,p.235.

6)Loc.Cit.

7)Le support signifiant renvoie aux marqueurs de nature lexicale qui permettent de distinguer les présupposés.

KERBRAT-ORECCHIONI(C.),L'implicite,p.38.

8) Les présupposés sont "(...) toutes les informations qui,sans être ouvertement posées (...)sont cependant automatiquement entraînées par la formulation de l'énoncé,dans lequel elles se trouvent intrinsèquement inscrites,quelque soit la spécificité du cadre énonciatif." ibid,p.25. 
renvoie à l'obséquiosité antérieure du beau-père et marque,par la suite, son caractère hypocrite.Cet adverbe accentue le décalage entre l'attitude d'auparavant/d'aujourd'hui et contribue à l'évolution de la dynamique polémique .

Dans un mouvement opposé à sa belle-fille,Iouri tente de se justifier.Cependant,sa tentative est avortée par son fils qui lui coupe brutalement la parole.En effet,Joe lance à son père un nouveau coup injonctif pour le rappeler à l'ordre:"-Tais-toi,je n'ai pas fini"1.1 Il le manipule et lui dénie le droit même de remplir activement son tour de parole.Ce qui souligne l'arrogance de Joe qui ne supporte pas de se voir contesté et exige d'être obéi au doigt et à l'œil.

Loin de se soumettre à la tyrannie de Joe,Mary sort de son silence pour réfuter le présupposé de son énoncé:"-Pardon,il ne voulait pas de toi." ${ }^{\prime 2} \mathrm{La}$ réprobation présuppose une proposition dont la vérité est susceptible d'être partagée par les interactants:"Joe a été favorablement accueilli par son beau-père".Mary a voulu rappeler à son mari la différence de leur statut et bouleverser le pouvoir que lui confère sa richesse.

Cette réfutation est "considérée comme plus polémique" ${ }^{\prime 3}$ que celle concernant le posé "en ce qu'elle vise moins l'énoncé que le cadre énonciatif posé par L1(locuteur1)"'t.

Ceci est d'autant plus mis en relief par l'intonation froide de Mary et par "Pardon".Ce rituel d'excuse fait ici office d'un régulateur ${ }^{5}$ signalant la la mise en cause de l'énonciation de Joe.Celui-ci affecte de se ranger du côté de sa femme.Il met en jeu un argument de communauté ${ }^{6}, " O n$ dit

1)JELINEK(H.),Op.Cit,p.82.

2)ibid,p.38.

3)NEVEU(F.),Phrases.Syntaxe,Rythme,Cohésion du texte,Paris,Éd.Sedes,1999,p.125.

4)Loc.Cit.

5)Les régulateurs sont des" signaux d'écoute" produits par les allocutaires pour garantir la poursuite de l'échange discursif.

KERBRAT-ORECCHIONI(C.),Les interactions verbales.Approche interactionnelle et structure des conversations, TI,p.18.

6)"La famille des arguments de communauté fait appel à des croyances et à des valeurs partagées par l'auditoire,qui contiennent déjà,en quelque sorte,l'opinion qui est l'objet de l'entreprise de conviction."

BRETON(PH.),l'argumentation dans la communication,Paris,Éditions La Découverte,2001 (Nouvelle édition),p.43.

7)JELINEK(H.), Op.Cit,p.38. 
$\varsigma a^{\prime \prime 1}$. Cependant, cet accord préalable perd son caractère concessif:il est déclaré sur un ton polémique,"Éclat de rire insultant de Joe",et contesté par le "mais" argumentatif.

Le cas est différent avec Iouri.Joe ne recourt pas au feint acquiescement ${ }^{2}$.Il s'élève directement contre son père et lit le rapport concernant John Ford "d'une voix de fonctionnaire qui rend son rapport..$^{\prime \prime 3}$ Ce qui témoigne de la détérioration des relations entre le père et le fils.Nous avons donc l'impression que les interactants progressent plutôt sur l'axe vertical ${ }^{4}$.L'affection s'est reculée et a fait place à l'hostilité l'hostilité et au conflit.

Nous pouvons dire que ce rapport constitue un argument de communauté par lequel Joe a voulu ridiculiser l'attitude de son père et légitimer ${ }^{5}$,par conséquent, son attaque.Devenu le père d'un des grands puissants de la société américaine,Iouri s'auto-dégrade en fréquentant un exterminateur.Il se déclare,de la sorte,inapte à s'intégrer à la Haute société et couvre son fils d'opprobre.

Joe accentue la gravité de son attaque par des cinétiques menaçants:"Joe se recule ...Il donne une claque sèche au papier ${ }^{\prime \prime}$

Pour mieux étayer son argument,il adresse à son père un reproche implicite sous la forme d'une assertion exclamative:"Un tueur de rats, et tu t'encanailles avec $\varsigma a !^{\prime \prime 7}$.Les exclamations ,qui parcourent le discours des interlocuteurs, révèlent leur"fort investissement émotif ${ }^{\prime \prime}$.

Face à ces coups successifs, Iouri devient éberlué.Il se sent complètement désarmé.Ne pouvant plus supporter la tension dramatique à laquelle il assiste,il cherche à fuir son malheur en pensant à Tony ${ }^{9}$ comme comme le donne à lire le discours attributif.

1)La concession ironique ou le feint acquiescement est un des procédés auquel recourent les interlocuteurs dans les épisodes polémiques pour combattre les arguments des uns et des autres.

NEVEU(F.),Op.Cit,p.130.

2)JELINEK(H.),Op.Cit,p.82.

3)Selon KERBRAT-ORECCHIONI(C.),il y a trois axes relationnels:l'axe horizontal ou l'axe de la familiarité,l'axe affectif et l'axe vertical "axe de la domination,ou du système des"places" KERBRAT-ORECCHIONI(C.),Les interactions verbales,TII,p.35.

4)Ceci s'explique par le fait que la justification de la violence "repose sur une argumentation acceptable socialement"

PRUD'HOMME(D.), "La violence à l'école n'est pas un jeu d'enfant",Paris,les éditions du remue-ménage,2006,p.50.

5) JELINEK(H.),Op.Cit,p.82.

6)ibid,p.83.

7)DURRER(S.),Op.Cit,p.90.

8)Tony était le voisin de Iouri à chicago.Il"(...)avait peur des bêtes des égouts."

JELINEK(H.),Op.Cit,p.40. 
Néanmoins,Mary tient tête à Joe et poursuit sa stratégie défensive.Elle procède à une disqualification de son discours à travers une interrogation négative orientée vers une réponse par"si". Mais,Joe ferme les yeux sur la valeur dérivée injonctive de cette "question orientée"11 et la considère comme une demande véritable d'informations.Il affiche son désaccord à Mary et confirme la négation par "non".Ainsi parvient-il à retourner la question de sa femme à son avantage et à garder sa position de force dans la mesure où :

"De manière générale, la question rhétorique est censée placer le questionneur en position haute:la réponse polémique opère alors une inversion de ce rapport de forces. ${ }^{2}$

Pour briser la résistance de sa femme rebelle,Joe lui profère des menaces vives dans des répliques successives.

Joe se lance également avec impétuosité à l'offense de son père.Il ne compatit pas à la souffrance du vieil homme qu'il a réduit à l'impuissance.Il lui assène une nouvelle injonction sous une forme interrogative:"Tu ...merde?"13.

Pour comble de malheur,Joe coupe court à la tentative de justification de son père.En effet,il a voulu tuer dans l'œuf toute contre-argumentation et obliger son père à faire amende honorable. À cette fin,il renvoie son père au banc des accusés et lui demande directement de déclarer les secrets qu'il a dévoilés à John Ford.

Mais,pour Iouri,affronter Joe est au dessus de ses forces.Il se sert donc de la dénégation afin de se garer de la fureur de son fils.Joe tend à son tour,à faire pression sur son père.Il le bourre d'une accusation de mensonge suivie d'une interrogation injonctive qui résonne avec sa question directe.

Iouri ,qui ne trouve pas d'échappatoire,finit par avouer le motif de son évasion.Joe récuse le posé de l'assertion de son père par "reformulation du positif en négatif ${ }^{\prime \prime}$.Il devient enragé et flagelle Iouri de ses assertions évaluatives négatives:accusations de trahison,de non-respect,de divulgation des secrets familiaux et de haine.La répétition du verbe"détester" met en relief l'écart affectif qui tranche le père et le fils.La mise en italique de l'adverbe interrogatif,"pourquoi",produit un

1)Il s'agit "(...)de question "orientée",plus précisément d"'interrogation rhétorique", lorsque la valeur assertive de la question est très nette."

NEVEU(F.),Op.Cit,p.117.

2)ibid,p.127.

3)JELINEK(H.),Op.Cit,p.83.

4)La reformulation du positif en négatif et celle du négatif en positif constituent deux types d'enchaînements non préférentiels caractérisant les épisodes polémiques.

NEVEU(F.),Op.Cit,p.124. 
effet d'insistance qui marque l'escalade de la dynamique polémique.Cette violence verbale de Joe s'est accentuée par sa violence physique:"Joe a posé ses deux mains...son regard accuse $e^{\prime 1}$.

Joe ne se comporte pas de façon moins agressive à l'égard de sa femme.Celle-ci tourne en dérision les menaces de son mari et répond par une exclamation, ,-Oh,que tu m'ennuies!"2, qui se fait plus caustique par son intonation,"Voix mondaine de Mary".Joe hurle des injures contre sa femme.Ce qui marque un tournant dans l'épisode.Il y a un changement du sujet de l'affrontement des interlocuteurs.Alors qu'au début de l'épisode,la dispute porte sur l'attitude du père de Mary envers son beau-fils et son ami,avec la progression discursive et actionnelle,elle concerne les interlocuteurs eux-mêmes:

"C'est souvent dans le passage de la réfutation des arguments à la récusation des intervenants eux-mêmes que procède la dynamique polémique (...)"13

Mary s'évertue à rendre le coup à Joe.Elle stigmatise l'attitude de son beau-père en déclarant qu'il la contemple avec convoitise au bord de la piscine.

Parallèlement,l'épisode J/I s'oriente vers le désaccord qui envenime la relation entre le fils et le père.Ce dernier veut affirmer son amour paternel.Ainsi tente-t-il d'infirmer le posé de l'interrogation de Joe, "pourquoi me détestes-tu?",, par un mouvement antithétique,(aimer/ détester), souligné par le "mais" argumentatif.Joe riposte par la reprise de l'énoncé $^{5}$ de Iouri qu'il investit d'ironie.L'itération du verbe"aimer" marque son opposition et son indignaton.

Iouri cherche à éclairer la situation à son fils voire à le ramener à la raison.Il lui explique que la richesse, autour de laquelle il gravite, est devenue une malédiction qui a rendu leur vie infernale. Joe bouleverse l'argument de son père en ayant recours à la reformulation du positif en négatif,"je n'en meurs pas ${ }^{\prime \prime}$.Il déclare que la richesse est ,pour lui, source

1)JELINEK(H.),Op.Cit,p.83.

2)ibid,p.39.

3)DURRER(S.),Op.Cit,pp.89-90.

4)JELINEK(H.),Op.Cit,p.83.

5)Les reprises des constituants de l'énoncé représentent une forme des enchaînements non préférentiels:"Ces reprises polémiques peuvent venir appuyer différentes formes de désaccord,selon l'acte illocutoire accompli dans l'intervention initiative."

NEVEU(F.),Op.Cit,p.123.

6)JELINEK(H.),Op.Cit,p.83. 
source de bonheur et de fierté. La valeur agonale de cette réfutation est mise en relief par le reproche implicite signalé par la reprise du verbe"mourir" mis en italique,"Peut-être... tu dis"ll. Le fait de "se taire brusquement" montre également qu'au cours de l'épisode,Joe a attaqué son père avec une violence telle qu'il s'est arrêté pour reprendre le souffle.

Les interlocuteurs sont donc en train d'échanger des répliques ayant des orientations argumentatives $^{2}$ de loin différentes.Ce qui révèle le divorce de leur univers de croyances:tandis que l'un adopte un raisonnement intellectuel basé sur la logique matérielle,l'autre prône l'amour et les valeurs humaines.Cette conception divergente de la vie est génératrice du désaccord qui dresse le fils contre le père.

Ce désaccord fait écho à la frustration que chaque membre du couple Joe/Mary éprouve à l'égard de l'autre.À sa femme ,qui a diffamé son père,Joe répond par une contre-attaque.Il l'accuse de mensonge.Mary ne peut plus se contenir.Elle se met hors de soi et va jusqu'à insulter son beau-père.Joe lui rend son insulte.Cet échange des propos désobligeants met à nu la haine qui déchire Joe/Mary et rend,par la suite,leur séparation inévitable ${ }^{3}$.

À l'instar de Mary,Iouri explose.Il reprend ses forces,"(...)je me redresse sur mon lit $^{\prime \prime}$, et lance une diatribe contre son fils.Il le désigne par des axiologiques négatifs:"un miteux,un nouveau riche" et lui crie son exaspération à travers des modalités interrogatives qui servent sa contre-argumentation.La répétition de la proposition,"Tout le monde a peur de to ${ }^{\prime \prime}$, dénote l'indignation de Iouri tourmenté par l'arrogance de Joe qui se plaît à écraser les autres.

Nous avons l'impression qu'il va y avoir un réajustement des rapports de force.Mais en vain! Joe interrompt son père par un rire si blessant qu'il décide d'exercer son autorité paternelle et de mettre fin à son effronterie en le battant:"je vais le battre"

Joe fait preuve d'une agressivité sans précédent.Il rend coup pour coup et arrête son père.Cette confrontation physique des interlocuteurs montre

\footnotetext{
1)JELINEK(H.),Op.Cit,p.84.

2)CHARAUDEAU(P.),MAINGUENEAU(D.),Op.Cit,p.493.

3)Joe et Mary se sont divorcés.

4) JELINEK(H.),Op.Cit,p.84.

5)Loc.Cit.

6)Loc.Cit.
} 
que la dynamique polémique a atteint son paroxysme.L'épisode s'achève sur la rupture entre Iouri $^{1}$ et son fils qui profère une mise en garde implicite,"Tu as ... père" ${ }^{\prime 2}$, et le quitte.

Le même manège se répète dans l'épisode $\mathrm{J} / \mathrm{M}$ qui débouche sur un point de non-retour.Après l'échange des injures,Joe ,affolé, "a dî envoyer sa chaussure,elle tape sur une porte déjà refermée" ${ }^{\prime \prime}$ et Mary s'est enfuie de la chambre.

De la sorte,Joe ouvre et ferme les épisodes.Ce qui prouve l'empire qu'il a sur sa femme et surtout sur son père.En effet,Joe est parvenu à étouffer la révolte de Iouri et à briser sa défense.Il a également provoqué sa femme qui lui a répondu avec une intensité égale.

L'affrontement des interactants a donc été à la base des deux épisodes et s'est manifesté dans les différentes répliques conflictuelles.La coordination argumentative de ces répliques est assurée par les réactions des interlocuteurs qui conviennent à la situation discursive:

"(...)deux énoncés $E_{1}$ et $E_{2}$ sont argumentativement coordonnés si le discours présente $E_{1}$ comme pouvant appuyer ou infirmer $E_{2}$ ou une conclusion favorisée par $E_{2} .^{14}$

Ce qui donne le jour à "l'auto et l'hétéro-continuité" suite,"la pertinence ${ }^{\prime \prime 6}$ des épisodes.Cette pertinence aboutit à la complétude interactionnelle qui consiste à considérer "(...)le discours comme une suite des interventions qui se complètent" ${ }^{7}$.

C'est la complétude interactionnelle qui concrétise la visée illocutoire globale des épisodes,et garantit,par conséquent, la cohérence discursive.

\footnotetext{
1)Iouri finit par devenir moine dans un couvent orthodoxe.

2)JELINEK(H.),Op.Cit,p.84.

3)ibid,p.40.

4)ANSCOMBRE(J.-C),DUCROT((C.),Op.Cit, p.117.

5)Tandis que l'auto-continuité est la "cohérence entre les différentes interventions successives de $L_{1}$ ou de $L_{2}(\ldots)$ ",l'hétéro-continuité est la "cohérence entre interventions produites consécutivement par deux locuteurs différents(...)".

KERBRAT-ORECCHIONI(C.),Les interactions verbales.Approche interactionnelle et structure des conversations, TI,p198.

6)"Un énoncé est d'autant plus pertinent qu'il produit plus d'effets et nécessite moins

d'efforts(cognitifs ou de traitement)".

SIOUFFI(G.),RAEMDONCK(D.-V),Op.Cit,p.183.

7)BAYLON(C.),MIGNOT(X.),La communication,Paris,Éd.Nathan,1997,p.200.
} 
Au cours de notre étude,nous avons essayé de montrer que la cohésion et la cohérence se complètent pour constituer l'armature discursive des épisodes.

Les différents moyens de la cohésion resserrent les liens syntactico-sémantiques sur le niveau inter/intra-répliques.L'endophore anaphorique/cataphorique contribue à consolider le tressage sémantique des répliques.L'articulation de ces répliques est favorisée par la progression thématique qui alterne les thèmes et les rhèmes et assure,par conséquent,la continuité discursive.

Pour faire prévaloir leur discours,les interlocuteurs recourent aux connecteurs qui contribuent à promouvoir leur stratégie défensive.Cette stratégie est mise en œuvre à travers l'isotopie, génératrice des termes révélateurs de l'antagonisme qui sépare Joe,sa femme et son père.

De la sorte,l'isotopie,les parenthésages,la progression thématique et l'endophore anaphorique/cataphorique aboutissent à la concaténation des répliques qui garantit l'intelligibilité des épisodes.

C'est la cohérence qui donne corps à la visée illocutoire globale de ces épisodes conformément à leur nature polémique.En effet, les interlocuteurs se sont engagés dans un va-et-vient discursif agonal.Ils se sont attaqués à travers les multiples assertions/contre-assertions qui témoignent de leurs divergences affectifs et ontologoiques.Chacun a essayé de surpasser l'autre et de bouleverser ses arguments.Ce qui a étouffé toute tentative de réconciliation et mené à la rupture entre les interlocuteurs.

Bref, Jelinek est parvenue à révéler les déchirements de ses protagonistes avec une telle dextérité qu'elle appelle à explorer d'autres domaines surtout celui de la psycholinguistique. 
Bibliographie 


\section{I.Corpus:}

\section{$\operatorname{JELINEK}(\mathrm{H}$.$) :$}

- Le Destin de Iouri Voronine,Paris,Éd. de Fallois,2005.(Grand Prix du roman de l'Académie française)

\section{II.L'R'uvre de JELINEK(H.):}

\section{***Romans:}

- La Vache multicolore,Paris,Éd.Gallimard,1961.

-Le Gentil Liseron,Paris,Éd.Gallimard,1963.

-La Route du wisky,Paris,Éd.Gallimard,1964.

-Portrait d'un séducteur,Paris,Éd.Gallimard,1965.

- La marche du fou,Paris,Éd.Gallimard,1967.

-La Vie de famille,Paris,Éd.Gallimard,1969.

-Les bêtes n'aiment pas l'amour des hommes,Paris,Éd.Gallimard,1972.

-Dans la nuit des deux mondes,Paris,Éd.Gallimard,1975.

-Ann Lee rachète les âmes,Paris,Éd.Julliard,1978.

-L'adolescente,Paris,Éd.Albatros, 1979.

-Le Porteur de Dieu,Paris,Éd.Julliard,1979.

-Madame le Président de la République française,Paris,Éd.Stock,1981.

-Une goutte de poison,Paris,Éd,Ramsay,1987.

\section{III.Ouvrages théoriques:}

**Ouvrages linguistiques:

-ADAM(J.M):

*Éléments de linguistique textuelle.Théorie et pratique de l'analyse textuelle,Bruxelles-Liège,Éd.Mardaga,1990(Deuxième édition).

*Les textes:types et prototypes,Paris,Éditions Nathan,1992(Troisième édition revue et corrigée).

*Linguistique textuelle.Des genres de discours aux textes,Paris,Édition Nathan,1999.

-ANSCOMBRE(J.-C),DUCROT(O.),L'argumentation dans la langue, Bruxelles,Éd.Mardaga,1983.

-BAYLON(C.),MIGNOT(X.),La communication,Paris,Éd.Nathan,1997. -BRETON(PH.), l'argumentation dans la communication, Paris,Éditions La Découverte, 2001(Nouvelle édition). -CALAS(F.),CHARBONNEAU(D.R),Méthode $d u \quad$ commentaire stylistique, Paris,Éditions Nathan,2000.

-DUCROT(O.),Les mots du discours,Paris,Les Éditions de Minuit,1980. -DURRER(S.),Le dialogue dans le roman,Paris,Éd.Nathan Université , 1999 . 
-KERBRAT-ORECCHIONI(C.):

* Les interactions verbales.Approche interactionnelle et structure des conversations,T.I, Paris, Éd.Armand Colin/Masson,1998(Troisième édition).

* Les interactions verbales,T.II,Paris,Éd.Armand Colin,1992.

* La conversation,Paris,Éditions Du Seuil,1996.

*L'énonciation de la subjectivité dans le langage,Paris,Éd.Armand Colin/Masson,1997(Troisième édition).

* L'implicite,Paris,Éd.Armand Colin,1998(Deuxième édition).

* Les actes de langage dans le discours. Théorie et fonctionnement, Paris,Éditions Nathan,2001.

-KLEIBER(G.),Anaphores et pronoms,Belgique,Éd.Duculot,1994.

-LÉVY(M.),PERSEC(S.), Grammaire du fransais.Approche énonciative, Paris,Éditions OPHRYS, 2000.

-MAINGUENEAU(D.):

*Nouvelles tendances en analyse du discours,Paris,Éd.Hachette, 1987.

*Éléments de linguistique pour le texte littéraire,Paris,Éd.Bordas, 1990(Nouvelle édition).

*Analyser les textes de communication,Paris,Éd.Nathan,2000.

-MOESCHLER(J.):

*Modélisation du dialogue.Représentation de l'inférence argumentative, Paris , Éd.Hermès, 1989.

*Théorie pragmatique et pragmatique conversationnelle ,Paris, Éd.Armand Colin/Masson,1996.

-NEVEU(F.),Phrases.Syntaxe,Rythme,Cohésion du texte,Paris, Éd.Sedes, 1999.

-PRUD'HOMME(D.),La violence à l'école n'est pas un jeu d'enfant, Paris,les éditions du remue ménage,2006.

-SIOUFFI(G.),RAEMDONCK(D.V),100 fiches pour comprendre la linguistique,Paris,Éd.Bréal,Rosny,1999.

-TISSET(C.),Analyse linguistique de la narration,Paris,Éd.Sedes, 2000.

-VION(R.),La communication verbale.Analyse des interactions,Paris,

Éd.Hachette, 1992.

\section{IV.Dictionnaires:}

-Petit Larousse en couleurs, Dictionnaire encyclopédique,Paris,

Éd.Larousse, 1991.

-Le Robert Mini.Langue française et noms propres,Paris,

Éd. Dictionnaires LE ROBERT,1995. 
-CHARAUDEAU(P.),MAINGUENEAU(D.),Dictionnaire d'analyse $d u$ discours,Paris,Éd. Du Seuil,2002.

\author{
V.Webiographie: \\ -"Biographie": \\ http://www.linternaute.com/biographie/henriette-jelinek/biographie/ \\ consulté le 17/03/2009. \\ -"Le Destin de Iouri Voronine": \\ http://www.alapage.com/-/Fiche/Livres/9782848681160/LIV/le-destin- \\ de-iouri-voron... consulté le 29/03/2009. \\ -"La critique [evene]": \\ http://www.evene.fr/livres/livre/henriette-jelinek-le-destin-de-iouri- \\ voronine-16235.php consulté le 17/03/2009.
}


Annexes 


\section{**L'épisode J/I:}

"1-Je ne dors pas,moi,et toi non plus,tu ne dormiras pas!

Il s'approche du lit:

2-Les Russes aiment la comédie,faire beaucoup de théâtre mais,avec moi,ça ne marche pas.Et je suis venu te le dire.

3-Joe...

4-Tais-toi,je n'ai pas fini.

Il sort un papier de sa poche,lit d'une voix de fonctionnaire qui rend son rapport:

5-John Ford,soixante-neuf ans,célibataire,né à Tulsa,Texas,venu en 1950 en Californie,établi à San Francisco puis Las Vegas,puis Los Angeles.Habite actuellement Venice.Une seule profession:exterminateur.

Joe se recule comme si j'allais lui apporter quelque bête tuée par John Ford.Il donne une claque sèche au papier.

6-Bref mais clair,non?Un tueur de rats, et tu t'encanailles avec $\varsigma$ a!

Je pense à Tony l'Italien de Chicago,je ne peux penser qu'à lui.Est-ce que Joe l'a connu?

7-Tu veux m'écouter,oui ou merde?

8-Mais...

9-Qu'est-ce que tu lui as raconté?

10-Rien.

11-Tous les Russes sont des menteurs,tu lui as raconté,dis-moi ce que tu lui as dit?

12-Que je voulais partir d'ici!

13-Ce n'est ce qu'il m'a dit!

Joe a posé ses deux mains sur le fauteuil,en face de mon lit,tend son buste vers moi et son regard accuse:

14-Tu m'as trahi,tu n'as aucun respect pour ton fils,tu divulgues toutes nos histoires de famille à de la racaille,tu cours le risque de nous amener quelques criminels,mais n'est-ce pas,qu'est- ce que $̧$ a fait?Tu me détestes à ce point,mais dis-le,que j'entende et qu'on en finisse!Alors, pourquoi me détestes-tu?

15-Mais je t'aime ,Joe.

16-Tu m'aimes,tu dis que tu m'aimes,mais comment tu m'aimes?

17-Tu ne comprends donc pas que cette richesse m'écoeure,non,ce n'est pas ca que je veux dire,je l'accepte ta richesse mais pourquoi on en meurt tous?

18-Mais moi, je n'en meurs pas, je suis content,fier.Peut-être que si tu en avais fait autant,je pourrais me reposer,je ne mourrais pas,comme tu dis. 
Il se tait brusquement comme s'il attendait une réponse.Est-ce que c'est son silence qui m'aide ou alors est-ce que j'ai un peu de courage?Je ne veux plus m'arrêter,je me redresse sur mon lit,les couvertures montrent l'encyclopédie,mais je m'en fous et je crie:

19-Tu ne seras jamais um prince mais un miteux, un nouveau riche qui nous en fiche plein la vue,qui regarde le caca qu'il fait,et on doit admirer,mais pourquoi?Qu'est-ce que tu fais de beau?Tout le monde a peur de toi,tu es content d'asservir,d'écraser,tout le monde a peur de toi,tout le monde cherche à te tromper,à profiter de toi et tu me fais pitié et j'ai honte..

Un éclat de rire m'interrompt,tellement insultant que je me lève,je vais le battre.Il m'arrête des deux bras tendus, me tient loin de lui puis me lâche. 20-Tu as de la chance d'être mon père."

Il est parti. 


\section{**L'épisode $\mathbf{J} / \mathbf{M}$ :}

"Mary est déjà dans sa chambre.Joe va la rejoindre directement. J'entends tout:

"1-Ton père m'a refusé son aide.Avec lui,on pouvait faire sortir Neal de taule,et tu sais ce que m'a répondu ce bol de merde?"La loi est la loi.De quoi aurais-je l'air?De protéger un homme présumé encore criminel?"Le sinistre con,il est content,il m'a blessé,il me prend pour un pauvre mec,mais qu'il fasse attention ton père!

Pas de réponse.

2-Il ne crânait pas autant lorsqu'il fallait te marier.

Voix froide de Mary:

3-Pardon,il ne voulait pas de toi.

Éclat de rire insultant de Joe:

4-On dit ça,mais il frétillait,le salaud.

Voix contenue de Mary:

5-Tu ne pourrais pas employer une autre expression?

6-Non,c'est la mienne.

Je n'entends plus rien puis vient la voix froide de Joe:

7-Mais je peux le briser ton père,tu entends,je peux le faire cimenter,et tu attendras longtemps pour le retrouver,car tu ne le retrouveras jamais.

Plus que méchant:

8-Ton prof de biologie ira étudier la vie des vers sous une dalle de béton.

Voix mondaine de Mary:

9-Oh,que tu m'ennuies!

On entend un grand coup sourd.

10-Nom de dieu,espèce de putain,ce n'est pas mon fric qui t'ennuie!Tu sais ce que tu es?

11-Un bol de merde.

Je l'entends marcher,elle doit être près de la porte.

12-Si mon père est ce que tu dis,le tien est un vieux vicieux qui aime trop mes seins et mon derrière.Mais oui,ne me regarde pas comme ça!Viens le voir installé derrière la porte-fenêtre,la bave aux lèvres.

Moi la bave aux lèvres.

13-Menteuse.

Mary a perdu le contrôle d'elle-même.

14-Ce vieux dégoûtant!

15-Dégueulasse toi-même.

Joe a dû envoyer sa chaussure,elle tape sur une porte déjà refermée.Mary s'en va dormir dans une des chambres du haut,le bruit de ses pas monte l'escalier." 